\title{
SOME REMARKS ON HOMEOMORPHISMS OF COMPACT LIE GROUPS
}

\author{
S. C. BAGCHI AND A. SITARAM
}

Dedicated to the memory of Irving Glicksberg

\begin{abstract}
It is proved that if $G$ is a compact connected semisimple Lie group and $H$ a compact group of homeomorphisms of $G$ containing all left and right translations of $G$, then there exists a positive integer $k$ such that for any $\tau \in H, \tau^{k}$ is, modulo a translation, an inner automorphism.
\end{abstract}

1. Introduction. In 1960 I. Glicksberg [2] proved the following result: Let $G$ be a compact connected abelian group. If $H$ is a compact group of homeomorphisms of $G$ containing all translations of $G$, then every element of $H$ is, modulo a translation, an automorphism. If moreover $G$ is finite dimensional, then the automorphisms involved are periodic.

This note is an attempt to generalize this result to nonabelian compact groups. However, we are forced to make two assumptions: (a) $G$ is a Lie group; and (for technical reasons) (b) $G$ is semisimple. Even with these assumptions one cannot get such a strong result as Glicksberg's theorem-why this is so will be clear from $\S 3$. What we essentially prove is that if $G$ is a compact connected semisimple Lie group and $H$ a compact group of homeomorphisms of $G$ containing all left and right translations of $G$, then there exists an integer $k$ such that for any $\tau \in H, \tau^{k}$ is, modulo a translation, an inner automorphism.

Finally we would like to mention that very little seems to be known about similar questions when $G$ is noncompact-even in the abelian case. However, if $G=\mathbf{R}^{h}$ some interesting results have been proved in [1] by Z. Charzyński.

2. Notation and preliminaries. For any unexplained notation and terminology see $[5,6$, and 7].

If $G$ is a Lie group, we denote by $G_{0}$ the connected component of $G$ containing the identity. By a metric $d$ on $G$, we mean a metric which defines the same topology as the given one on $G$. On the group of homeomorphisms of $G$ we impose the compact-open topology and by a "compact group of homeomorphisms of $G$ " we mean a subgroup of the group of homeomorphisms on $G$ which is also a compact subset in the compact open topology. By (left translation) $l_{g}$ and (right translation)

Received by the editors December 13, 1983.

1980 Mathematics Subject Classification. Primary 22E99, 53C35; Secondary 54H15.

Key words and phrases. Semisimple Lie group, Riemannian symmetric space, compact transformation group. 
$R_{h}$ we mean the homeomorphisms of $G$ defined by: $l_{g}(x)=g x, R_{h}(x)=x h, x \in G$. If $M$ is a manifold, then $Q$ will denote a Riemannian structure on $M$ and $I^{Q}(M)$ the corresponding group of isometries (with respect to $Q$ ) of $M$.

If $G$ is a compact (Lie) group, $\hat{G}$ will denote the set of equivalence classes of irreducible (finite dimensional) representations of $G$. If $\delta \in \hat{G}$, let $\pi \in \delta$ and let $V$ be the vector space on which $G$ acts via the representation $\pi$. Set $d(\delta)=\operatorname{dim} V$. (Note that this is well defined.) If $v_{1}, v_{2} \in V$ consider the continuous function on $G$ defined by $x \rightarrow\left(\pi(x) v_{1}, v_{2}\right)$, where $($,$) is some inner product on V$. Such a function is called "a matrix element" corresponding to $\delta$. A finite linear combination of matrix elements corresponding to $\delta$ will be called "a representative function of class $\delta$ ". By a "representative function" on $G$ we mean a function of the form $\sum_{i=1}^{k} f_{i}$, where each $f_{i}$ is a representative function of class $\delta_{i}$. Let $V_{\delta}$ be the subspace of $C(G)$ consisting of those $f$ for which $\xi_{\delta} * f=f$. Here * denotes convolution in the group $G$ and $\chi_{\delta}$ is the 'character' corresponding to $\delta$ (see [7]). (Each $V_{\delta}$ is finite dimensional and in fact $\operatorname{dim} V_{\delta}=(d(\delta))^{2}$ and if $f$ is a "representative function" on $G$, then $f \in V_{\delta_{1}}+V_{\delta_{2}}+$ $\cdots+V_{\delta_{k}}$ for some $\delta_{1}, \delta_{2}, \ldots, \delta_{k} \in \hat{G}$.) We now record an observation which follows essentially from the Peter-Weyl theorem and the fact that if $G$ is a Lie group then any representative function is smooth (and in fact real analytic).

ObSERVATION 2.1. If $G$ is a compact Lie group and $\tau: G \rightarrow G$ is a homeomorphism, then $\tau$ is a diffeomorphism if for each representative function $f, f \circ \tau$ is also a representative function.

We now record some theorems which will be used in §3. These are taken from [5] and [6].

THEOREM 2.2. (See the theorem on p. 244 of [6].) If a compact group $H$ acts effectively on a connected locally Euclidean space $E$ and if all the orbits in $E$ are locally connected, then $H$ is a Lie group.

THEOREM 2.3. (See the theorem on p. 212 of [6].) Let $H$ be a Lie group which acts as a transformation group on a manifold of class $C^{k}$ (analytic) and assume that for each $g \in H, x \mapsto g \cdot x$ is of class $C^{k}$ (analytic) in $x$. Then $(g, x) \mapsto g \cdot x$ is of class $C^{k}$ (analytic) in $g$ and $x$ simultaneously.

TheOREM 2.4. (See Theorem 4.1 on p. 207 in [5].) Let $(G, K)$ be a Riemannian symmetric pair. Suppose that $G$ is semisimple and acts effectively on the coset space $M=G / K$. Let $Q$ be any $G$-invariant Riemannian structure on $M$. Then $G=I_{0}^{Q}(M)$ (as Lie groups).

Now let $G$ be a compact, connected Lie group and let $\Delta=\{(g, g) ; g \in G\} \subseteq G$ $\times G$. Then one can identify $G$ with the Riemannian symmetric pair $(G \times G, \Delta)$. This observation coupled with Theorem 2.4 immediately yields

COROLlARY 2.5. Let $G$ be a compact, connected, semisimple Lie group and $Q$ a bi-invariant (i.e. left and right invariant) Riemannian structure on $G$. Then if $\tau \in$ $I_{0}^{Q}(G)$, then $\tau=R_{g} \circ l_{h}$ for some $g, h \in G$.

Finally if $V$ is a Banach space and $\left\{V_{i}\right\}$ is a family of closed subspaces of $V$, by $V=\oplus \sum V_{i}$ we mean that the $\left\{V_{i}\right\}$ form an algebraic direct sum which is dense in $V$. 
We are now in a position to state and prove the results mentioned in the introduction.

3. The main results. We start with the following proposition.

Proposition 3.1. Let $G$ be a compact connected Lie group and $H$ a compact group of homeomorphisms of $G$ containing all left translations of $G$. Then:

(i) Every element $\tau$ of $H$ is a diffeomorphism of $G$.

(ii) There exists a left invariant Riemannian structure $Q$ on $G$ such that $H \subseteq I^{Q}(G)$.

COROllary 3.2. Let $G$ be a compact connected Lie group and $d$ a left invariant metric on $G$. ( $d$ need not necessarily arise from a Riemannian structure.) Let $\phi$ be an isometry of $G$ with respect to $d$. Then $\phi$ is a diffeomorphism and is in fact an isometry of $G$ with respect to some left invariant Riemannian structure $Q$ on $G$.

Proof of Proposition 3.1. Since $H$ can be considered as a compact transformation group on $G, H$ acts on $C(G)$ in a natural way and by the Peter-Weyl theorem $C(G)=\oplus \sum H_{i}$, where the $H_{i}$ are minimal $H$-invariant subspaces. Moreover each $H_{i}$ is finite dimensional and since $H$ contains all left translations of $G$, each $H_{i}$ is also $G$-invariant under the left regular action. Thus each $H_{i}$ can be written $\oplus \sum_{k=1}^{n(i)} H_{i k}$ and $H_{i k}$ is a minimal $G$-invariant subspace and hence $C(G)=\oplus \sum_{i=1}^{\infty} \sum_{k=1}^{n(i)} H_{i k}$ is a decomposition of $C(G)$ into minimal left $G$-invariant subspaces. Thus it follows that if $\delta \in \hat{G}$, then $V_{\delta} \subseteq H_{i_{1}} \oplus \cdots \oplus H_{i_{l}}$ for some $i_{1}, \ldots, i_{l}$. If $f$ is a representative function of class $\delta$, then $f \in V_{\delta}$ and hence for any $\tau \in H, f \circ \tau \in H_{i_{1}} \oplus \cdots \oplus H_{i_{i}}$. (Note also that for any $i$ every function in $H_{i}$ is a representative function.) Thus by Observation 2.1, $\tau$ is a diffeomorphism. This proves part (i) of the proposition and now Theorems 2.2 and 2.3 of $\$ 2$ imply that $H$ is a compact Lie transformation group on $G$ and hence by a standard (Haar) integration argument we can construct an $H$-invariant Riemannian structure $Q$ on $G$. Since $H$ contains all left translates of $G$, part (ii) of the proposition follows.

The corollary is immediate if we take $H$ to be the group of isometries of $G$ with respect to $d$. (Note that by Ascoli's theorem $H$ will be compact.)

We are now in a position to state and prove our main theorem.

THEOREM 3.3. Let $G$ be a semisimple, compact, connected Lie group and $H a$ compact group of homeomorphisms of $G$ containing all left and right translations of $G$. Then:

(i) Every element $\tau$ of $H$ is a diffeomorphism of $G$.

(ii) There exists a bi-invariant Riemannian structure $Q$ of $G$ such that $H \subseteq I^{Q}(G)$.

(iii) There exists a positive integer $k$ such that if $\tau \in H$, then $\tau^{k}=l_{h} \circ R_{g}$ for some $g \in G, h \in G$. (Thus if moreover $\tau(e)=e, \tau^{k}$ is an inner automorphism of $G$.)

Proof of Theorem 3.3. The first two parts follow from Proposition 3.1, when we now take into account that $H$ contains both left and right translations. Since $I^{Q}(G)$ is a compact Lie group, $I^{Q}(G) / I_{0}^{Q}(G)$ is finite and hence $\exists k$ such that $\forall \tau \in H$, $\tau^{k} \in I_{0}^{Q}(G)$. Since $G$ is semisimple, the last part of the theorem now follows from Corollary 2.5 of $\S 2$. 
COROllary 3.4. Let $G$ be a semisimple, compact, connected Lie group and $d a$ bi-invariant metric on $G$. ( $d$ need not necessarily arise from a Riemannian structure.) Let $\phi$ be an isometry of $G$ with respect to $d$. Then $\phi$ is a diffeomorphism and moreover:

(i) $\phi$ is an isometry with respect to some bi-invariant Riemannian structure $Q$ on $G$.

(ii) There exists an integer $k$ such that $\phi^{k}=l_{h} \circ R_{g}$ for some $g \in G, h \in H$.

The corollary follows from the theorem if we take $H=$ group of isometries of $G$ with respect to $d$.

An application. In [3] Glicksberg proves (using results from [2]) that if $f$ is a continuous function on a compact connected abelian group and $\phi$ is any homeo of $G$ such that ${ }^{x} f \circ \phi={ }^{x^{\prime}} f$ for all $x \in G$, then $\phi=T \circ l_{g}$ for some automorphism $T$ of $G$ and $g \in G$. Further if $G$ is finite dimensional, then $T$ is periodic. In the spirit of the above we prove

THEOREM 4.1. Let $G$ be a semisimple compact connected Lie group. Let $f$ be a continuous function on $G$ such that $x \mapsto^{x} f$ is 1-1 and $f$ is $\operatorname{Ad} G$ invariant. If $\phi$ is a homeomorphism of $G$ such that ${ }^{x} f \circ \phi$ is a left translate of $f\left(={ }^{x} f\right.$ say) for all $x \in G$, then there exists an integer $k$ such that $\varphi^{k}=l_{g} \circ R_{h}$ for some $g, h \in G$. (Thus if $\varphi(e)=e, \phi^{k}$ is an inner automorphism.)

Proof. Define a metric $d$ on $G$ by $d(x, y)=\left\|^{x} f-{ }^{y} f\right\|_{\infty}$. Then using the Ad-invariance of $f$, we can show that $d$ is a bi-invariant metric on $G$ and exactly as in [3], $\phi$ is an isometry with respect to this metric. The theorem now follows from Corollary 3.4 .

REMARK. Theorem 4.1 would still be valid if we only assume $f \in L^{P}(G)$ but then we would also have to assume $\phi$ preserves Haar measure on $G$.

We conclude with some remarks that generalize the results in $\S 3$.

5. Concluding remarks. Actually the methods of $\$ 3$ can be used to prove the following remarks about symmetric spaces of the compact type: Let $X$ be a symmetric space of the compact type, $I(X)$ its group of isometries and $G$ the connected component containing $e$ of the group of isometries of $X$. (Then $X$ can be identified with $G / K$ for a suitable subgroup $K$ of $G$.) If $d$ is any $G$-invariant metric on $X$ (defining the topology of $X$ ), then there exists a $G$-invariant Riemannian structure $Q$ on $X$ such that $I^{d}(X) \subseteq I^{Q}(X)$. But now $I_{0}^{Q}(X)=I_{0}(X)=G$ and hence there exists $k$ such that for any $\phi \in I^{d}(X), \phi^{k} \in G$. If we take $X=S^{n-1}$, the standard $n$-sphere in $\mathbf{R}^{n}$, then $I(X)=O(n), G=S O(n)$ and it is not too hard to show that in this case $I^{Q}(x) \subseteq O(n)$. Thus if $d$ is any rotation invariant metric on $S^{n-1}$ and $\phi$ is an isometry of $S^{n-1}$ with respect to $d$ then actually $\phi$ is given by an element in $O(n)$. These observations for $S^{n-1}$ and more generally for rank-1 symmetric spaces have in fact been already made by Glicksberg - see the discussion following Theorem 6 in [4].

In conclusion we would like to point out that we can in fact make the following even more general remark: If $G$ is a compact connected Lie group and $K$ a closed subgroup of $G$ and $d$ is any $G$-invariant metric on $G / K$, then there exists a $G$-invariant Riemannian structure $Q$ on $G / K$ such that $I^{d}(G / K) \subseteq I^{Q}(G / K)$. The case $K=\{e\}$ is really Proposition 3.1 (and Corollary 3.2). 
Acknowledgments. The authors would like to express their thanks to Dr. R. Gangolli for his interest in this work. The crucial use of [6] in this paper was suggested by him. The authors also thank Dr. S. G. Dani and Dr. Gopal Prasad for several useful discussions on isometries of compact groups and symmetric spaces. Finally we would like to record our gratitude to the late Dr. I. Glicksberg for his encouragement and interest in this work.

\section{REFERENCES}

1. Z. Charzyński, Sur les transformations isometriques des espaces du type (F), Studia Math. 13 (1953), 94-121.

2. I. Glicksberg, Some special transformation groups, Proc. Amer. Math. Soc. 11 (1960), 315-318.

3. __ Maps preserving translates of a function, Pacific J. Math. 87 (1980), 323-334.

4. Transition operator characterizations of compact and maximally almost periodic locally compact groups, preprint.

5. S. Helgason, Differential geometry and symmetric spaces, Academic Press, New York, 1962.

6. D. Montgomery and L. Zippin, Topological transformation groups, Interscience, New York, 1966.

7. L. S. Pontryagin, Topological groups, Gordon \& Breach, New York, 1966.

Stat - Math Division, Indian Statistical Institute, Calcutta 700 035, India

Department of Mathematics, University of Washington, Seattle, Washington 98195 